\title{
A RECURRENT NONROTATIONAL HOMEOMORPHISM ON THE ANNULUS
}

\author{
ROBBERT J. FOKKINK AND LEX G. OVERSTEEGEN
}

\begin{abstract}
We construct an area- and orientation-preserving recurrent diffeomorphism on the annulus without periodic points, which is not conjugate to a rotation. The mapping is, however, semiconjugate to an irrational rotation of a circle. Our example is a counterexample to the Birkhoff Conjecture.
\end{abstract}

\section{INTRODUCTION}

Periodic maps on the annulus were classified in the 1950's by Kerékjártó [Ker], who showed that they are conjugate to a rotation or reflection about the diameter (modulo a flip of the boundaries). This result was extended a few years later by Hemmingson [Hem] to almost periodic homeomorphisms. The example presented here shows that Hemmingson's result cannot be generalized to recurrent homeomorphisms. However, due to a result of Oversteegen and Tymchatyn [OT], if a recurrent homeomorphism on the annulus has a periodic point, then it is a periodic map.

Various authors have referred to the following as the Birkhoff Conjecture (see in particular [Mar, Hal]):

Birkhoff Conjecture. Suppose $h: \mathbf{A} \rightarrow \mathbf{A}$ is an area- and orientation-preserving homeomorphism on an annulus without periodic points. Then $h$ is conjugate to an irrational rotation.

The only reference to this problem which we have been able to find in the works of Birkhoff carries the additional hypothesis that $h$ is analytic [Bi2, Problem 15]. If $h$ is complex analytic, the desired result follows, since $h$ can be extended to a Möbius transformation of the Riemann sphere by the Schwarz reflection principle. It remains an open question whether there are real analytic examples.

Our example is closely related to examples of Handel and Herman. Herman [Her] has constructed a $C^{\infty}$-diffeomorphism $h$ of the plane, with the pseudocircle $\mathbf{P}$ (a nonlocally connected, hereditarily indecomposable, circularly chainable continuum) as a minimal continuum, such that $h$ is complex analytic and complex analytically conjugate to an irrational rotation $r_{\alpha}$ on the

Received by the editors August 8, 1990 .

1980 Mathematics Subject Classification (1985 Revision). Primary 58F99, 28D05; Secondary $54 \mathrm{H} 20$.

Key words and phrases. Recurrent, area preserving, annulus, almost periodic.

The second author was supported in part by NSF-RII-8610669. 
bounded complementary domain of $\mathbf{P}$ and $C^{\infty}$-conjugate to $r_{\alpha}$ on the unbounded complementary domain. Handel [Han] produced an earlier example with $\mathbf{P}$ as a minimal set, but with weaker properties. Straightforward modifications of Herman's example, using for instance a theorem of Oxtoby and Ulam (which states that absolutely continuous measures on a disc are topologically equivalent) to obtain measure preservation, will yield a counterexample to the Birkhoff Conjecture as stated above. Thus we do not claim that our example represents the first solution to this problem. The main difference between the diffeomorphism constructed in this paper and the one constructed by Herman is that the hairy circle, which is invariant under our diffeomorphism, is simpler than the pseudocircle. Indeed the hairy circle allows the diffeomorphism to be semiconjugate to a rotation (it was shown in [Han] that the restriction to $\mathbf{P}$ is not semiconjugate to a rotation) and to be recurrent. The results in this paper were announced in [FO].

As pointed out by Fathi [Fa] it is possible to show the existence of an areapreserving recurrent diffeomorphism on the annulus with a dense orbit, following methods developed by Anosov and Katok [AK] (see also [FH]). The argument used is similar to Oxtoby's proof $[\mathrm{O}, \mathrm{p}$. 70] of the existence of an area-preserving homeomorphism of the disk with a dense orbit.

We are indebted to Edward Tymchatyn for many helpful discussions and suggestions. In particular, his conviction that the hairy circle admitted a recurrent homeomorphism encouraged us to study this possibility and gave rise to the results obtained here.

We would also like to thank Sebastian van Strien who pointed our attention to the Birkhoff conjecture and made some valuable remarks, which improved the exposition of our example.

\section{DEFINITIONS AND PRELIMINARIES}

We will denote the natural numbers by $\mathbf{N}$, the integers by $\mathbf{Z}$, the set of real numbers by $\mathbf{R}$, and the annulus by $\mathbf{A}=\mathbf{S}^{1} \times[0,1]$. In general we will make no distinction between $\mathbf{S}^{1}$ and the unit interval $\mathbf{I}=[0,1]$ with identified end points.

A homeomorphism $h: X \rightarrow X$ on a compact metric space $X$ is periodic provided $h^{n}=\mathrm{id}_{X}$ for some $n \in \mathbf{Z}$, and almost periodic provided for each $\varepsilon>0$ there exists a relatively dense set $R_{\varepsilon} \subset \mathbf{Z}$ (i.e., there exists $N>0$ such that for each $\left.n \in \mathbf{Z},[n, n+N] \cap R_{\varepsilon} \neq \varnothing\right)$, such that for each $n \in R_{\varepsilon}$, $d\left(h^{n}, \operatorname{id}_{X}\right)<\varepsilon$, where $d$ is the standard complete metric on the space of homeomorphisms of $X$. It is well known [Go] that a homeomorphism is almost periodic iff the collection of iterates $\left\{h^{n}\right\}$ forms an equicontinuous family iff there exists an equivalent metric on $X$ in which $h$ is an isometry. The last condition is often used to show the existence of invariant simple closed curves for nonperiodic, almost periodic homeomorphisms on plane continua as a first step towards conjugating the homeomorphism with a rotation. Plane continua with interior which admit almost periodic (nonperiodic) homeomorphisms were completely classified by Hemmingson [Hem]. He showed they are either discs or annuli and the almost periodic homeomorphisms are essentially rotations. A homeomorphism $h: X \rightarrow X$ is called recurrent provided, for each $\varepsilon>0$, there exists $n \in \mathbf{Z}$ such that $d\left(h^{n}, \mathrm{id}_{X}\right)<\varepsilon$. Recurrent homeomorphisms on 
discs or annuli with a periodic point on the boundary are periodic [OT] and hence completely classified. Clearly periodic $\Rightarrow$ almost periodic $\Rightarrow$ recurrent and easy examples show that none of these implications can be reversed.

\section{THE HAIRY CIRCLE}

We will first construct a simple homeomorphism on the annulus which is not conjugate to a rotation. This homeomorphism will be of the form

$$
H(x, r)=\left(r_{\alpha}(x), f(x, r)\right)
$$

for some irrational rotation $r_{\alpha}$ on $\mathbf{S}^{1}$. In order to prevent the homeomorphism from being conjugate to a rotation we push an interior point to the boundary, i.e., the orbit closure of this point contains the inner boundary circle. ${ }^{1}$ Imagine that the point is connected to the inner boundary by an arc $J$. Suppose that the images of $J$ under iterates of the recurrent homeomorphism $h$ form a set of disjoint arcs. On one hand there are arbitrarily small arcs since the end point of $J$ is pushed to the boundary. On the other hand there must be long arcs since $h$ is a recurrent homeomorphism. The union of the inner boundary circle and the set of images of $J$ form an invariant set. In this way we obtain a topological space which we call a hairy circle. The hairy circle is an invariant continuum under $h$. Our strategy is to give an explicit construction of this invariant continuum first and then investigate what homeomorphisms it allows.

Construction of the hairy circle. Let the circle be the unit interval with identified end points. Points on the circle are represented in the standard binary expansion. For each point $x=\left(x_{1}, x_{2}, \ldots\right) \in \mathbf{S}^{\mathbf{1}}, x_{i} \in\{0,1\}$, partition $\left\{x_{1}, x_{2}, \ldots\right\}$ into consecutive pairs $B_{j}(j \geq 1)$. Hence, $B_{1}=\left\{x_{1}, x_{2}\right\}$, $B_{2}=\left\{x_{3}, x_{4}\right\}$, and in general $B_{j}=\left\{x_{2 j-1}, x_{2 j}\right\}$. Let

$$
\psi_{j}(x)=\left|2\left(1-x_{2 j-1}-x_{2 j}\right)\right| \text {. }
$$

In other words, $\psi_{j}(x)$ is the absolute value of the difference between the number of 0 's and the number of 1's in the $j$ th pair. Let $\Psi(x): \mathbf{S}^{1} \rightarrow(0, \infty]$ and $l(x): \mathbf{S}^{1} \rightarrow[0,1)$ be defined by

$$
\Psi(x)=\sum_{j=1}^{\infty} \frac{\psi_{j}(x)}{j} \text { and } l(x)=2^{-1-\Psi(x)} .
$$

Note that for each point on the circle which does not have a unique binary representation, there exists an $n$ such that $x_{i}=x_{j}$ for $i, j \geq n$, i.e., the binary expansion has a constant tail. The set of elements with constant binary tail is a dense subset of the circle. For every element $x$ with constant tail $\Psi(x)=\infty$ and $l(x)=0$. Put

$$
\mathbf{H}=\left\{(\theta, r) \in \mathbf{A} \mid \theta \in \mathbf{S}^{1} \text { and } 0 \leq r \leq l(\theta)\right\} .
$$

$\mathbf{H}$ is called the hairy circle, and the arc of length $l(x)$ at $x \in \mathbf{S}^{1}$ is called the hair at $x$. We claim that $\mathbf{H}$ is a one-dimensional nonlocally connected

\footnotetext{
${ }^{1}$ We are indebted to Fathi for pointing out an alternative way of pushing a point to the boundary, using techniques inspired by [AK] (see also [FH]), due to Herman: Let $C$ be the space of all areapreserving $C^{\infty}$ diffeomorphisms of the annulus that are isotopic to the identity and let $D$ be the closure of the set $\left\{h^{-1} \circ r_{\alpha} \circ h \mid h \in C\right\}$, where $r_{\alpha}$ is the rigid rotation of the annulus by $\alpha$. It can be shown that the subset of $D$ consisting of recurrent diffeomorphisms with a dense orbit is a dense $G_{\delta}$ in $D$.
} 
continuum. The one-dimensionality of $\mathbf{H}$ and the nonlocally connectedness are obvious from the observation that the elements with constant tail have no hair attached. We have to prove that $\mathbf{H}$ is compact. Let $\left(x_{n}\right)$ be a sequence in $\mathbf{S}^{1}$ converging to $x$. Then

$$
\liminf _{n \rightarrow \infty} \sum_{j=1}^{\infty} \frac{\psi_{j}\left(x_{n}\right)}{j} \geq \sum_{j=1}^{N} \frac{\psi_{j}(x)}{j}
$$

for every $N \in \mathbf{N}$. This implies that $\Psi$ is lower semicontinuous, therefore $\mathbf{H}$ is compact. Note that the set of elements $x$ in $\mathbf{S}^{1}$ with nondegenerate hairs is a dense $F_{\sigma}$ since $\left\{x \in \mathbf{S}^{1} \mid l(x) \geq \frac{1}{n}\right\}$ is a closed set with empty interior. Also, each nondegenerate hair is a limit "from two sides" of other hairs of $\mathbf{H}$. It follows from results in [BO] or [Ch] that with this property $\mathbf{H} / \mathbf{S}^{1}$ is topologically unique. Recently, Aarts and Oversteegen [AO] proved that the hairy circle itself is topologically unique (indeed, between any two hairy circles in the plane there exists a homeomorphism which can be extended to the plane).

\section{A NONROTATIONAL HOMEOMORPHISM ON THE ANNULUS}

We have constructed the hairy circle in such a way that it is likely that it admits recurrent homeomorphisms without periodic points. First we show that there exist periodic homeomorphisms on the hairy circle arbitrarily close to the identity map. A suitable composition of periodic homeomorphisms indeed converges to a recurrent homeomorphism without periodic points on the hairy circle. Then we indicate how these periodic homeomorphisms can be extended to the annulus. By taking a limit of the extended homeomorphisms we obtain the desired nonrotational homeomorphism $H$. We indicate how to modify this construction such that this homeomorphism $H$ is in addition recurrent and conjugate to an irrational rotation in the complement of $\mathbf{H}$. In this case one can define an invariant measure $\left(C^{0}\right.$-conjugate to the Lebesgue measure) on the annulus (see [OU]). It is not difficult to see that the hairy circle $\mathbf{H}$ cannot be invariant under a diffeomorphism. However, it is possible to change the lengths of the hairs in such a way that a homeomorphic copy of $\mathbf{H}$ is invariant under a $C^{\infty}$-diffeomorphism. Our original construction [FO] was in fact along these lines. That this is possible follows from a simplification of the results in $\S 5$. This way we will obtain a recurrent $C^{\infty}$-diffeomorphism of the annulus which preserves the radial foliation, has no periodic points and is not conjugate to a rotation. Since the Oxtoby-Ulam Theorem [OU] has no smooth analog, this construction is limited to invariant measures which are $C^{0}$-conjugate to the Lebesgue measure. In the next section we will improve on these results and obtain a diffeomorphism which preserves the Lebesgue measure. However, in this case the diffeomorphism does not keep the radial foliation of the annulus invariant. We will first show the existence of arbitrary small periodic homeomorphisms on $\mathbf{H}$ :

1. Lemma. For every $n \in \mathbf{N}$ there exists a homeomorphism $h_{n}: \mathbf{H} \rightarrow \mathbf{H}$ of period $2^{n}$ such that

$$
\lim _{n \rightarrow \infty} d\left(h_{n}, \mathrm{id}\right)=0
$$


Proof. The periodic rotation $r_{n}$ of the circle is defined by

$$
r_{n}(x)=\left(x+\frac{1}{2^{n}}\right) \quad \bmod 1 .
$$

Observe that $r_{n}(x)$ differs from $x$ only in the first $n$ coordinates of the binary expansion of $x$. In other words,

$$
\text { if } x=\sum_{i=1}^{\infty} \frac{x_{i}}{2^{i}} \text {, then } r_{n}(x)=\left(\sum_{i=1}^{n} \frac{x_{i}}{2^{i}}+\frac{1}{2^{n}}\right)+\sum_{i=n+1}^{\infty} \frac{x_{i}}{2^{i}}(\bmod 1) \text {. }
$$

Partition the circle into $2^{n}$ intervals $\left\{J_{1}^{n}, J_{2}^{n}, \ldots, J_{2^{n}}^{n}\right\}$ having at most end points in common such that any two points in one of these intervals have the same first $2^{n}$ coordinates. If we assume that $n$ is even, then $r_{n}$ permutes these intervals cyclically. Moreover, for each $j$ there exists a constant $c_{j}^{n}$ such that $l(x)=c_{j}^{n} \cdot l\left(r_{n}(x)\right)$ for each $x \in J_{j}^{n}$. As a result we can linearly extend the rotation $r_{n}$ to the hairy circle; $h_{n}: \mathbf{H} \rightarrow \mathbf{H}$ is defined as $h_{n}(x, t)=\left(r_{n}(x), c_{j}^{n} \cdot t\right)$ for an element $(x, t) \in \mathbf{H}$ such that $x \in J_{j}^{n}$.

Observe that the $c_{j}^{n}$ do not converge to 1 as $n \rightarrow \infty$. However, as is shown below, the $c_{j}^{n}$ are only significant for those $j$ for which the length of the hairs, $l(x)$, is small.

In order to prove that the periodic homeomorphisms $h_{n}$ converge to id as $n \rightarrow \infty$, we first prove the following claim.

Claim. Let $n$ be an even integer and let $m$ be an integer such that $m<n$. Then $\max \left(c_{j}^{n}, 1 / c_{j}^{n}\right) \geq 2^{\frac{1}{m}}$ only if

$$
J_{j}^{n} \subset\left\{\left(y_{i}\right) \mid y=\sum_{i=1}^{\infty} \frac{y_{i}}{2^{i}} \text { with } y_{i}=1 \text { for } 2 m-1 \leq i \leq n\right\} .
$$

Observe that addition of $\frac{1}{2^{n}}$ afflicts the first digit $x_{1}$ in the binary expansion $\left(x_{1}, x_{2}, \ldots\right)$ only if the digits $x_{i}$ equal 1 for every $2 \leq i \leq n$. In general we get the following equalities:

$$
l(y): l\left(r_{n}(y)\right)=2^{-\Psi(y)}: 2^{-\Psi\left(r_{n}(y)\right)}=2^{-\sum_{j=1}^{\infty} \psi_{j}(y) / j}: 2^{-\sum_{j=1}^{\infty} \psi_{j}\left(r_{n}(y)\right) / j}
$$

by the definition of $\Psi$. Since the addition of $\frac{1}{2^{n}}$ afflicts only the first $n$ digits, we observe that

$$
2^{-\sum_{j=1}^{\infty} \psi_{j}(y) / j}: 2^{-\sum_{j=1}^{\infty} \psi_{j}\left(r_{n}(y)\right) / j}=2^{-\sum_{j=1}^{n / 2} \psi_{j}(y) / j}: 2^{-\sum_{j=1}^{n / 2} \psi_{j}\left(r_{n}(y)\right) / j} .
$$

Suppose that the first digit to alter under the addition of $\frac{1}{2^{n}}$ is an element of the $k$ th pair $\left\{x_{2 k-1}, x_{2 k}\right\}$. Then all pairs from the $(k+1)$ st to the $\frac{n}{2}$ th are equal to $\{1,1\}$. Therefore addition of $\frac{1}{2^{n}}$ alters these pairs into $\{0,0\}$, which does not change the difference between the numbers of zero's and the number of ones.

$$
2^{-\sum_{j=1}^{n / 2} \psi_{j}(y) / j}: 2^{-\sum_{j=1}^{n / 2} \psi_{j}\left(r_{n}(y)\right) / j}=2^{-\psi_{k}(y) / k}: 2^{\psi_{k}\left(r_{n}(y)\right) / k} \leq 2^{1} / k .
$$

The above claim implies that $\left|l(x)-c_{j}^{n} \cdot l(x)\right| \rightarrow 0$ if $n \rightarrow \infty$. Indeed, if $\max \left(c_{j}^{n}, 1 / c_{j}^{n}\right) \geq 2^{\frac{1}{m}}$ for a very large $n$, then the elements of $J_{j}^{n}$ have to contain a very long sequence of pairs $\{0,0\}$. In other words, the length $l(x)$ 
of elements $x \in J_{j}^{n}$ is small: for every $\varepsilon>0$ there exists an $n \in \mathbf{N}$ such that $l(x)<\varepsilon$ for all $x \in J_{j}^{n}$ if $\left|c_{j}^{n}-1\right| \geq \varepsilon$. Therefore the periodic homeomorphisms $h_{n}$ converge to the identity map if $n \rightarrow \infty$.

Remark. The homeomorphisms $h_{n}$ commute.

2. This follows from the observation that the $h_{n}$ map hairs linearly onto hairs. In order to obtain a recurrent homeomorphism $h$ on $\mathbf{H}$ without periodic points, we can take the infinite composition of a subsequence $h_{i(n)}$ :

$$
h=\cdots \circ h_{i(n)} \circ \cdots \circ h_{i(1)} \circ h_{i(0)} .
$$

Every finite composition $\rho_{n}=h_{i(n)} \circ \cdots \circ h_{i(0)}$ is a periodic homeomorphism on $\mathbf{H}$, since the $h_{n}$ commute. In order to obtain a recurrent homeomorphism $h$ in the limit, we must choose $h_{i(n+1)}$ such that the infinite composition converges sufficiently fast.

As we see, it is possible to construct a recurrent nonperiodic homeomorphism on the hairy circle without periodic points. Now we want to extend this homeomorphism to the annulus, or rather we want to extend the $h_{n}$ to the annulus. This turns out to be very simple. It requires more care to extend the $h_{n}$ in such a way that the limit homeomorphism $H$ is recurrent.

3. Lemma. There exists a sequence $H_{n}$ of periodic homeomorphisms on the annulus $\mathbf{A}$, such that:

(1) $H_{n}(x, t)=\left(r_{n}(x), p_{n}(x, t)\right)$ with $t \rightarrow p_{n}(x, t)$ piecewise linear.

(2) $H_{n}$ has period $2^{n}$.

(3) $d\left(H_{n}, \mathrm{id}_{\mathbf{A}}\right) \rightarrow 0$ as $n \rightarrow \infty$.

(4) $H_{n} \mid \mathbf{H}=h_{n}$.

It is possible to adapt the construction of the $H_{n}$ in such a way that the limit $H=\cdots \circ H_{i(n)} \circ \cdots \circ H_{i}(1) \circ H_{i}(0)$ is recurrent.

Proof. We will only outline the construction. As defined above $h_{n}: \mathbf{H} \rightarrow \mathbf{H}$ is a periodic homeomorphism of period $2^{n}$ of the form

$$
h_{n}(x, t)=\left(r_{n}(x), c_{j}^{n} \cdot t\right),
$$

where $r_{n}(x)=\left(x+\frac{1}{2^{n}}\right) \bmod 1$ and $x \in J_{j}^{n}$. Define $\chi_{n}: \mathbf{S}^{1} \rightarrow[0,1)$ by

$$
\chi_{n}(x)= \begin{cases}\max \left\{l(y) \mid \frac{j}{2^{n}} \leq y \leq x\right\} & \text { if } x \in\left[\frac{j}{2^{n}}, \frac{j+\frac{1}{2^{n}}}{2^{n}}\right], \\ \max \left\{l(y) \mid x \leq y \leq \frac{j+1}{2^{n}}\right\} & \text { if } x \in\left[\frac{j+\frac{1}{2}}{2^{n}}, \frac{j+1}{2^{n}}\right] .\end{cases}
$$

Define the homeomorphism $H_{n}: \mathbf{A} \rightarrow \mathbf{A}$ such that, for each $x \in \mathbf{S}^{1}$, $H_{n} \mid\{x\} \times I:\{x\} \times I \rightarrow\left\{r_{n}(x)\right\} \times I$ maps the intervals $\left[0, \chi_{n}(x)\right]$ and $\left[\chi_{n}(x), 1\right]$ linearly onto the intervals $\left[0, \chi_{n}\left(r_{n}(x)\right)\right]$ and $\left[\chi_{n}\left(r_{n}(x)\right), 1\right]$, respectively. Since $\chi_{n} \circ r_{n}(x)=c_{j}^{n} \cdot \chi_{n}(x)$ for $x \in J_{j}^{n}, H_{n} \mid \mathbf{H}=h_{n}$.

Choose $m_{j}^{n} \in J_{j}^{n}$ such that $\chi_{n}(x) \leq \chi_{n}\left(m_{j}^{n}\right)$ for each $x \in J_{j}^{n}$. Note that $\left(m_{j}^{n}, \chi_{n}\left(m_{j}^{n}\right)\right) \in \mathbf{H}$. Then for each $x \in J_{j}^{n}$,

$$
d((x, t), H(x, t)) \leq d\left(\left(m_{j}^{n}, \chi_{n}\left(m_{j}^{n}\right)\right), H\left(m_{j}^{n}, \chi_{n}\left(m_{j}^{n}\right)\right) \leq d\left(h_{n}, \mathrm{id}_{\mathbf{H}}\right) .\right.
$$

Hence (3) follows from the fact that $d\left(h_{n}, \mathrm{id}_{\mathbf{H}}\right) \rightarrow 0$ as $n \rightarrow \infty$.

In this way we have extended the $h_{n}$ without taking care of the behavior on the complement of the hairy circle. Therefore we cannot expect to obtain 
periodic compositions $\rho_{n}=H_{i(n)} \circ \cdots \circ H_{i(0)}$. We indicate how this can be achieved.

By approximating the graphs $\chi_{i(n)}$ from above by the graphs of continuous functions $\gamma_{n}: \mathbf{S}^{1} \rightarrow(0,1)$ we obtain a sequence of descending simple closed curves. The extension $R_{i(n)}$ is the rigid rotation $r_{i(n)}$ on the first coordinate and it maps the intervals

$$
\left[0, \gamma_{n}(x)\right],\left[\gamma_{n}(x), \gamma_{n-1}(x)\right], \ldots,\left[\gamma_{1}(x), 1\right]
$$

linearly onto

$$
\left[0, \gamma_{n}\left(r_{i(n)}(x)\right)\right],\left[\gamma_{n}\left(r_{i(n)}(x)\right), \gamma_{n-1}\left(r_{i(n)}(x)\right)\right], \ldots,\left[\gamma_{1}\left(r_{i(n)}(x)\right), 1\right],
$$

respectively. Due to a similar argument as in the proof of Lemma 1, it can be shown that $R_{i(n)} \rightarrow \mathrm{id}_{\mathrm{A}}$ as $n \rightarrow \infty$. Although condition (4) does not hold, the infinite composition $H=\cdots \circ R_{i(n)} \circ \cdots \circ R_{i(1)} \circ R_{i(0)}$ maps hairs of $\mathbf{H}$ linearly onto hairs. Therefore it extends the recurrent, nonperiodic homeomorphism $h$ on the hairy circle. On the complement of $\mathbf{H}$ the map $H$ is conjugate to an irrational rotation on the half open annulus $\mathbf{S}^{1} \times(0,1]$. By choosing a suitable sequence $i(n)$ the extended $H$ is also recurrent.

We will indicate next how to obtain a $C^{\infty}$-diffeomorphism keeping the radial foliation of $\mathbf{A}$ (and a topological copy of $\mathbf{H}$ ) invariant. Since the construction is a simplification of the construction in $\S 5$, we will only formulate the necessary lemma and the theorem. The proof of Theorem 5 in this section is identical to the proof of Theorem 8 in the next section. One only needs to replace Corollary 7 by Lemma 4 in the argument. The proof of Lemma 4 is a straightforward application of a $C^{\infty}$ bump function and is omitted. We will refer to a simple closed curve of the form $S^{1} \times\{t\}$ as a round circle and to a rotation $\rho(x, t)=$ $(x+\alpha, t)$ (in polar coordinates) as a rigid rotation.

4. Lemma. Let $g: S^{1} \rightarrow(0,1)$ be a $C^{\infty}$-function such that the graph $K=$ $\left\{(x, g(x)) \mid x \in S^{1}\right\} \subset \mathbf{A}$ is invariant under a rigid rotation $\rho$. Then there exists a $C^{\infty}$-diffeomorphism $f: \mathbf{R}^{2} \rightarrow \mathbf{R}^{2}$ of the plane such that:

(1) $f \mid \mathbf{R}^{2} \backslash \mathbf{A}=\mathrm{id}_{\mathbf{R}^{2} \backslash \mathbf{A}}$.

(2) $f \circ \rho=\rho \circ f$ and $f$ keeps radial lines invariant.

(3) $f(K)$ is a round circle.

By repeating the argument for Theorem 8 (replacing the use of Corollary 7 by Lemma 4) one produces the recurrent diffeomorphism of Theorem 5 . It follows from results in [AO] that there exists a homeomorphism of the plane which maps the invariant continuum $B=\bigcap B_{n}$ (see Theorem 8) onto the hairy circle $\mathbf{H}$.

5. Theorem. There exists a recurrent $C^{\infty}$-diffeomorphism $H: \mathbf{A} \rightarrow \mathbf{A}$ keeping a homeomorphic copy of the hairy circle $\mathbf{H}$ invariant such that

$$
H(x, t)=(\rho(x), f(x, t))
$$

for an irrational rotation $\rho$ of $S^{1}$.

\section{THE AREA-PRESERVING CASE}

As remarked above, an application of a result by Oxtoby and Ulam [OU] yields an invariant measure for the diffeomorphism constructed in Theorem 5, 
which is $C^{0}$-conjugate to the Lebesgue measure. We would like to improve this result by constructing a recurrent $C^{\infty}$ diffeomorphism which is not conjugate to a rotation and is area-preserving in the Lebesgue sense (the Lebesgue measure is induced by $[0,1] \times[0,1])$. It is not easy to take the approach as outlined in the preceding section. First of all only a homeomorphic copy of the hairy circle, as defined in $\S 4.2$, is invariant under a diffeomorphism. A second obstruction is that the Oxtoby-Ulam theorem has no smooth analog. Therefore we take a different approach following Handel and Herman, see [Han] and [Her]: we construct a sequence of arbitrarily small perturbations, such that the composition converges to the desired diffeomorphism. Note that this procedure can not be applied to real analytic diffeomorphisms.

We need an elementary lemma, implicitly contained in [Han] and [Her]. The interior of an arc $K$ is the subset of non-end-points.

6. Lemma. Let $K$ be a $C^{\infty}$ embedded arc in the unit square $\mathbf{B}=[0,1] \times$ $[0,1] ; K$ separates the bottom $[0,1] \times\{0\}$ from the top $[0,1] \times\{1\}$ and the interior of $K$ is contained in $\mathbf{B}^{\circ}=(0,1) \times(0,1)$. There exists an areapreserving $C^{\infty}$ diffeomorphism $f: \mathbf{B} \rightarrow \mathbf{B}$ which maps $K$ onto a horizontal arc $[0,1] \times\{t\}$ for some $t \in[0,1]$.

Proof. In fact we prove the inverse statement and construct a diffeomorphism which maps a horizontal arc onto $K$. Let $\mathbf{B}_{1}$ and $\mathbf{B}_{2}$ be the two components such that $\mathbf{B}_{1} \cup \mathbf{B}_{2}=\mathbf{B}$ and $\mathbf{B}_{1} \cap \mathbf{B}_{2}=K$. Both $\mathbf{B}_{1}$ and $\mathbf{B}_{2}$ are diffeomorphic to the unit square. Therefore there exists a homeomorphism $\gamma: \mathbf{B} \rightarrow \mathbf{B}$ which maps a horizontal arc $C=[0,1] \times\{s\}$ onto $K$. On the complement of $C, \gamma$ is a diffeomorphism. According to [Hir, p. 182] the homeomorphism $\gamma$ can be isotoped to a diffeomorphism $\phi$ such that $\left.\phi\right|_{C}=\left.\gamma\right|_{C}$. We transform $\phi$ into an area-preserving diffeomorphism in two steps.

Let $\alpha(t)$ be the area of $\phi([0,1] \times[0, t])$. Obviously $\alpha:[0,1] \rightarrow[0,1]$ is a $C^{\infty}$ diffeomorphism. The diffeomorphism $\phi_{1}: \mathbf{B} \rightarrow \mathbf{B}$ is defined as

$$
\phi_{1}(x, y)=\phi\left(x, \alpha^{-1}(y)\right) \text {. }
$$

The area of a rectangle of the form $[0,1] \times[0, y]$ is preserved by $\phi_{1}$. In order to obtain an area-preserving diffeomorphism, the Jacobian has to equal 1 everywhere. Let $J: \mathbf{B} \rightarrow(0, \infty)$ be the Jacobian of $\phi_{1}$. The diffeomorphism $\phi_{2}: \mathbf{B} \rightarrow \mathbf{R}^{2}$ is defined as

$$
\phi_{2}(x, t)=\left(\int_{0}^{x}(J(y, t))^{-1} d y, t\right) .
$$

We have achieved that $f=\phi_{2} \circ \phi_{1}: \mathbf{B} \rightarrow \mathbf{R}^{2}$ is an area-preserving diffeomorphism. Its image is not a priori equal to $\mathbf{B}$. However, since $\phi_{1}$ preserves the area of $[0,1] \times[0, t]$ for all $t \in[0,1]$, so does $\phi_{2}$. This implies that $\phi_{2}$ preserves $\{1\} \times[0,1]$. We conclude that $f$ is a diffeomorphism on the unit square, and that it maps the arc $C$ onto $K$.

We apply this lemma to the annulus to obtain the following corollary. Like Lemma 6 , this corollary can also be stated for an arbitrary $C^{\infty}$-embedded simple closed curve which separates the boundary circles.

7. Corollary. Let $g: S^{1} \rightarrow(0,1)$ be a periodic $C^{\infty}$-function such that the graph $K=\left\{(x, g(x)) \mid x \in S^{1}\right\} \subset \mathbf{A}$ separates the boundary circles. If $K$ 
is invariant under a periodic rotation $\rho$, then there exists an area-preserving diffeomorphism $f$ on $\mathbf{A}$ such that $f(K)$ is a round circle $S^{1} \times\{t\}, t \in(0,1)$, and $f \circ \rho=\rho \circ f$.

Proof. Let $n$ be the period of $\rho$. The annulus can be divided into finitely many wedges $W_{1}, W_{2}, \ldots, W_{n}$ which are permuted by $\rho, W_{i}=\{(x, t) \in$ A| $\left.\frac{i-1}{n} \leq x \leq \frac{i}{n}\right\}$. According to Lemma 6 the arc $K \cap W_{i}$ can be mapped onto a horizontal arc for each $i$ by an area-preserving diffeomorphism $f_{i}$. Moreover, since $K$ is invariant under $\rho$, all $f_{i}$ are essentially equal. Finally, we claim that the $f_{i}$ can be chosen in such a way that $f_{i}=f_{i+1}$ on $W_{i} \cap W_{i+1}$. In other words, the diffeomorphism $f_{1}$ induces the same map on the left and right side of $W_{1}$. This follows from the proof of Lemma 6: the diffeomorphism $\phi$ can be chosen to induce the same map on the left and right side of the wedge $W_{1}$. In that case the union of all $f_{i}$ induces a diffeomorphism on the annulus which commutes with $\rho$.

If the hairy circle is approximated by simple closed curves, these curves have to be increasingly folded up and down in the annulus. Now, contrary to the construction in $\S 3$ we do not start out with an invariant continuum. In order to obtain an invariant continuum like the hairy circle, we construct a sequence of increasingly folded, periodic curves. This invariant continuum is a circle with continua attached. We denote by $d_{\infty}$ the complete metric on the space of $C^{\infty}$-diffeomorphisms.

8. Theorem. There exists a sequence $\psi_{n}$ of area-preserving $C^{\infty}$-diffeomorphisms on $\mathbf{A}$, a sequence $\rho_{n}$ of rigid rotations, and a sequence $K_{n}$ of $C^{\infty}$. embedded simple closed curves, such that:

(1) $\rho_{n}$ has period $p_{n}$ and for all $n \in \mathbf{N}, p_{n} \mid p_{n+1}$.

(2) $h_{n}=\psi_{n} \circ \rho_{n} \circ \psi_{n}^{-1}$ is a Cauchy sequence; $d_{\infty}\left(h_{n+1}^{i}, h_{n}^{i}\right)<\frac{1}{2^{n}}$ for all $1 \leq i \leq p_{n}$.

(3) $\psi_{n}$ maps a round circle onto $K_{n}$.

(4) $K_{n}$ divides $\mathbf{A}$ into two annuli $A_{n} \cup B_{n}=\mathbf{A}$ and $A_{n} \cap B_{n}=K_{n}$. The annulus $A_{n}$ contains the outer boundary $\mathbf{S}^{1} \times\{1\}$ and $K_{n+1} \subset B_{n}$.

(5) The maps $\psi_{n}^{-1}$ and $\psi_{m}^{-1}$ coincide on $A_{m}$ for $m \leq n$.

(6) The intersection $B=\bigcap\left\{B_{n} \mid n \in \mathbf{N}\right\}$ is a one-dimensional nonlocally connected continuum.

The limit diffeomorphism $h=\lim _{n \rightarrow \infty} h_{n}$ is a recurrent and area-preserving diffeomorphism. The continuum $B$ is invariant under $h$.

Proof. The proof is by induction. We choose the maps $\psi_{1}$ and $\rho_{1}$ to be the identity $\operatorname{id}_{\mathbf{A}}$ and $K_{1}$ is the circle $\mathbf{S}^{1} \times\left\{\frac{1}{2}\right\}$. Suppose that $\rho_{i}, \psi_{i}, K_{i}$ are defined for $1 \leq i \leq n$. Let $C_{n+1}$ be the graph of a periodic $C^{\infty}$-function in the interior of $\psi_{n}^{-1}\left(B_{n}\right)$ invariant under a rigid rotation of period $p_{n+1}$. The integer $p_{n+1}$ is a multiple of $p_{n}$ to be determined later. According to Corollary 7 there exists an area-preserving diffeomorphism $\psi: \mathbf{A} \rightarrow \mathbf{A}$ which maps $C_{n+1}$ to a round circle. Moreover we can choose $\psi$ to equal the identity on $\psi_{n}^{-1}\left(A_{n}\right)$ by applying the corollary to the annulus $\psi_{n}^{-1}\left(B_{n}\right)$. This diffeomorphism commutes with the rigid rotation of period $p_{n+1}$ on $\psi_{n}^{-1}\left(B_{n}\right)$. We define $\psi_{n+1}=\psi_{n} \circ \psi^{-1}$ and $K_{n+1}=\psi_{n}\left(C_{n+1}\right)$. The rigid rotation $\rho_{n+1}$ is the composition of $\rho_{n}$ and the rigid rotation $(x, t) \rightarrow\left(x+\frac{1}{p_{n+1}}, t\right)$. 
In order to satisfy condition (2) $p_{n+1}$ has to be chosen sufficiently large. We claim that

$$
\psi_{n+1} \circ \rho_{n+1} \circ \psi_{n+1}^{-1}=\psi_{n} \circ \rho_{n+1} \circ \psi_{n}^{-1} .
$$

The equality holds on the annulus $B_{n}$ since $\psi$ and $\rho_{n+1}$ commute. It holds on $A_{n}$ since $\psi$ is the identity on $\psi_{n}^{-1}\left(A_{n}\right)$. Therefore, by choosing $p_{n+1}$ sufficiently large the conditions (1) through (5) can be satisfied. In order to satisfy condition (6) we have to choose the curves $K_{n}$ increasingly folded. Notice that up till now we only required the curve $C_{n+1}$ to be the graph of a periodic function. The folding condition is as follows: (a) Divide the inner boundary circle into $p_{n}$ points $w_{1}=(0,0), w_{2}=\left(\frac{1}{p_{n}}, 0\right), \ldots, w_{p_{n}}=\left(1-\frac{1}{p_{n}}, 0\right)$. The periodic curve $C_{n+1}$ can be chosen such that $K_{n+1}$ contains $z_{1}, z_{2}, \ldots, z_{p_{n}}$ with $d\left(w_{i}, z_{i}\right) \leq \frac{1}{2^{n}}$ for all $1 \leq i \leq p_{n}$. (b) $d\left(K_{n+1}, \mathbf{S}^{1} \times\{1\}\right)<\frac{3}{4}$.

Condition (b) implies that $\bigcap\left\{B_{n} \mid n \in \mathbf{N}\right\}$ contains a point $y$ in the interior of A. According to condition (b) there exists a unique $x \in \mathbf{S}^{\mathbf{1}} \times\{0\}$ such that $x$ and $y$ are connected via a continuum $D \subset B$ with $D \cap\left[\mathbf{S}^{1} \times\{0\}\right]=\{x\}$. Since the limit diffeomorphism $h=\lim _{n \rightarrow \infty} h_{n}$ is recurrent and nonperiodic, $h^{n}(D)$ returns arbitrarily close to $D$ without intersecting it. Hence $\bigcap\left\{B_{n} \mid n \in \mathbf{N}\right\}$ is not locally connected. The description of the invariant continuum is not as explicit as in $\S 3$. Indeed, the continuum $D$ above may not be an arc.

We conclude with two questions:

(1) Does the set of admissible rotation numbers for recurrent diffeomorphisms as in Theorem 8 have full measure?

(2) Does there exist an area-preserving nonperiodic analytic diffeomorphism of the annulus which leaves a topological copy of the hairy circle invariant?

\section{REFERENCES}

[AO] J. M. Aarts and L. G. Oversteegen, The geometry of Julia sets in the exponential family (in preparation).

[AK] D. V. Anosov and A. B. Katok, New examples in smooth ergodic theory, Trans. Moscow Math. Soc. 27 (1972), 117-134.

[Bi1] G. D. Birkhoff, Proof of Poincaré's geometric theorem, Trans. Amer. Math. Soc. 14 (1913), 14-22.

[Bi2] __ Some unsolved problems of theoretical dynamics, Science 94 (1941), 598-600; Collected Mathematical Papers, vol. II, Amer. Math. Soc., Providence, R.I., 1950, pp. 710-712.

[Boy] P. Boyland, The rotation set as a dynamical invariant, preprint.

[BO] W. T. Bula and L. G. Oversteegen, A characterization of smooth Cantor bouquets, Proc. Amer. Math. Soc. 108 (1990), 529-534.

[Ch] W. J. Charatonik, The Lelek fan is topologically unique, Houston J. of Math. (to appear).

[Fa] A. Fathi, Private communication.

[FH] A. Fathi and M. Herman, Existence de difféomorphismes minimaux, Astérique 49 (1977), 37-59.

[Fo] N. E. Foland, A characterization of the almost periodic homeomorphisms on the closed 2-cell, Proc. Amer. Math. Soc. 16 (1965), 1031-1034.

[FO] R. J. Fokkink and L. G. Oversteegen, An example related to the Birkhoff conjecture, preprint, 1989.

[Got] W. H. Gottschalk, Minimal sets: An introduction to topological dynamics, Bull. Amer. Math. Soc. 64 (1958), 336-351. 
[Hal] G. R. Hall, Some problems on dynamics of annulus maps, Hamiltonian Dynamical Systems (Boulder, Col., 1987), Contemp. Math., vol. 81, Amer. Math. Soc., Providence, R.I., 1988, pp. 135-152.

[Han] M. Handel, A pathological area preserving $C^{\infty}$ diffeomorphism of the plane, Proc. Amer. Math. Soc. 86 (1982), 163-168.

[Hem] E. Hemmingson, Plane continua admitting non-periodic autohomeomorphisms with equicontinuous iterates, Math. Scand. 2 (1954), 119-141.

[Her] M. R. Herman, Construction of some curious diffeomorphisms of the Riemann sphere, J. London Math. Soc. 34 (1986), 375-384.

[Hir] M. W. Hirsch, Differential topology, Springer, New York, 1976.

[Ker] B. de Kerékjártó, Sur la structure des transformation topologiques des surfaces en ellesmèmes, Enseign. Math. 35 (1952), 297-316.

[Mar] L. Markus, Three unresolved problems of dynamics on surfaces, preprint.

[Mon] D. Montgomery, Remarks on groups of homeomorphisms, Amer. Math. Monthly 48 (1941), 310-317.

[OT] L. G. Oversteegen and E. D. Tymchatyn, Recurrent homeomorphisms on the plane are periodic, Proc. Amer. Math. Soc. (to appear).

[O] J. C. Oxtoby, Measure and category, Springer, Berlin, 1980.

[OU] J. C. Oxtoby and S. M. Ulam, Measure preserving homeomorphisms and metrical transitivity, Ann. of Math. (2) 42 (1941), 874-920.

[Si1] E. Sidorov, Smooth topologically transitive dynamical systems, Math. Notes Acad. Sci. USSR 4 (1968), 939-943.

[Si2] _ Topologically transitive cylindrical cascades, Math. Notes Acad. Sci. USSR 14 (1973), 810-816.

Delft University of Technology, Department of Pure Mathematics, P.O. Box 5031, 2600 Ga Delft, The Netherlands

Department of Mathematics, University of Alabama, Birmingham, Alabama 35294 